\title{
Bronchoskopie im Miniaturformat
}

\begin{abstract}
In der Humanmedizin sind endoskopische Lungenspiegelungen mittels Videobronchoskop gängige Praxis. Wissenschaftler der Charité Berlin haben nun ein Miniatur-Bronchoskop entwickelt. Für die experimentelle Lungenforschung ein entscheidender Schritt - künftig lassen sich deutlich genauere und aussagekräftigere Ergebnisse erzielen. Erste Anwendungen sind in der Fachzeitschrift American Journal of Respiratory Cell and Molecular Biologie Ende 2014 veröffentlicht worden.
\end{abstract}

Lungenerkrankungen gehören zu den häufigsten Erkrankungen des Menschen entsprechend zahlreich sind Forschungen auf dem Gebiet der Pneumologie. Um dabei wiederholte Untersuchungen an Atemwegen und Lunge zu ermöglichen, hat ein interdisziplinäres Team von Wissenschaftlern um Prof. M. Witzenrath und Prof. A. Meisel nun ein Bronchoskop in Miniaturform entwickelt. Es ist das kleinste bisher vorhandene Gerät und in Zusammenarbeit mit Experten eines spezialisierten Herstellers entstanden. Eine Herausforderung in der Lungenforschung bspw. an Mäusen ist die geringe Größe ihrer Atemsysteme. Mit der neuen Technik entwickeln die Wissenschaftler minimalinvasive Anwendungen für dieses Forschungsfeld. Genau lokalisierte Untersuchungen oder die gezielte Behandlung bestimmter Lungenregionen sind jetzt auf kleinstem Raum möglich. „Das Verfahren eröffnet neue Perspektiven in der Forschung und bessere Kontrollmethoden. Erstmalig lassen sich bspw. beide Lungenflügel getrennt voneinander behandeln und beobachten“, so Prof. Witzenrath. Somit können Vergleichsstudien oder Verlaufsuntersuchungen an ein und demselben Tier durchgeführt werden.

Die Bilder und Daten der fiberoptischen Miniaturbronchoskope können nach Angaben der Forscher künftig dazu beitragen, Krankheitsmechanismen bei Menschen mit Lungenerkrankungen besser zu verstehen. Gleichzeitig hilft die neue Methodik, die Zahl von Untersuchungstieren zu reduzieren.

Nach einer Mitteilung der Charité, Berlin 